\title{
Qualidade de vida de idosos submetidos à quimioterapia antineoplásica atendidos em um hospital de referência oncológica
}

\section{Quality of life of elderly patients submitted to antineoplastic chemotherapy treated at an oncology reference hospital}

Jessika Cardoso de Souza ${ }^{1,2}$, Emerson Glauber Abreu dos Santos³, Anderson Lineu Siqueira dos Santos², Maria Izabel Penha de Oliveira Santos², Daiane de Souza Fernandes ${ }^{3}$, Tatyellen Natasha da Costa Oliveira ${ }^{2,4}$

' Fundação Hospital de Clínicas Gaspar Vianna, Belém, Pará, Brasil

2 Universidade do Estado do Pará, Belém, Pará, Brasil

${ }^{3}$ Universidade Federal do Pará, Belém, Pará, Brasil

${ }^{4}$ Instituto Evandro Chagas, Ananindeua, Pará, Brasil

\begin{abstract}
RESUMO
OBJETIVOS: Avaliar a qualidade de vida $(Q V)$ dos idosos submetidos ao tratamento quimioterápico antineoplásico, assim como suas associações com fatores socioeconômicos, nível de dependência e dados clínicos. MATERIAIS E MÉTODOS: Realizou-se um estudo transversal com 200 idosos atendidos em um ambulatório de um hospital de referência oncológica do estado do Pará, Brasil. A QV foi avaliada pelo instrumento EORTC QLQ-C30 v3, constituído por 30 questões que abrangiam 15 domínios, divididos em três escalas distintas: escala de estado de saúde global e QV; escala funcional; e escala de sintomas. O nível de dependência foi avaliado pela Escala de Lawton e Brody, que permite analisar a capacidade do indivíduo em realizar as atividades instrumentais da vida diária, como utilizar o telefone, administrar o dinheiro, realizar compras, preparar refeições e viajar. Os dados foram analisados por meio de estatística descritiva, teste de qui-quadrado e ANOVA. RESULTADOS: Evidenciou-se uma QV mediana. As escalas saúde global e funcional apresentaram resultados medianos, enquanto a escala de sintomas demonstrou melhor $Q V$ em seus domínios. Identificou-se uma relação significante entre a QV dos idosos e o nível de dependência. Os domínios mais afetados foram dificuldade financeira, fadiga, desempenho de papéis e função social. CONCLUSÃO: A interferência da QV, bem como o nível de dependência dos idosos, estão associados à doença, seu tratamento e às condições de morbidade.
\end{abstract}

Palavras-chave: Qualidade de Vida; Idosos; Idoso Fragilizado; Antineoplásicos.

\begin{abstract}
OBJECTIVES: To evaluate the quality of life (QL) of elderly patients submitted to antineoplastic chemotherapy and its associations with socioeconomic factors, level of dependence, and clinical data. MATERIALS AND METHODS: A cross-sectional study was conducted on 200 elderly patients attending an outpatient clinic at a oncology reference hospital in Pará State, Brazil. QL was evaluated by the EORTC QLQ-C30 v3 instrument, consisting of 30 questions covering 15 domains, divided into three distinct scales: global health status scale and QL; functional scale; and scale of symptoms. The dependency level was evaluated by the Lawton and Brody Scale, which allows the analysis of the individual's ability to perform the instrumental activities of daily living, such as using the telephone, administering money, shopping, preparing meals, and traveling. Data were analyzed using descriptive statistics, chi-square test, and ANOVA. RESULTS: A median QL was demonstrated. The global and functional health scales presented median results, while the symptom scale showed better $Q L$ in its domains. A significant relation was identified between the QL of elderly and the level of dependence. The most affected domains were financial difficulty, fatigue, performance, and social function. CONCLUSION: QL interference and the level of dependence of elderly are associated with the disease, its treatment, and morbidity conditions.
\end{abstract}

Keywords: Quality of life; Elderly; Frail Elderly; Antineoplastic Agents.

\author{
Correspondência / Correspondence: \\ Tatyellen Natasha da Costa Oliveira \\ Instituto Evandro Chagas/SVS/MS \\ Rodovia BR-316 km 7, s/n. Bairro: Levilândia. CEP: 67030-000 - Ananindeua, Pará, Brasil - Tel.: +55 (91) 3214-2185 \\ E-mail: tatyellenoliveira@iec.gov.br
}




\section{INTRODUÇÃO}

$\bigcirc$ processo de envelhecimento da população e a alteração na estrutura da pirâmide etária do Brasil, nas últimas décadas, relacionam-se intrinsecamente com o crescimento socioeconômico, os avanços no campo da saúde pública e as mudanças no estilo de vida do brasileiro'. Consequentemente, observou-se uma alteração no perfil de morbimortalidade do país, evidenciado pela diminuição dos casos de doenças infectocontagiosas e aumento de doenças crônico-degenerativas ${ }^{2}$.

Nesse cenário de mudanças demográficas e epidemiológicas, as doenças crônico-degenerativas, como neoplasias, doenças circulatórias, respiratórias, ósseas, musculares e articulares, que afetam principalmente idosos, destacam-se por acarretarem elevados gastos aos serviços de saúde, além de interferirem na qualidade de vida (QV) das pessoas acometidas e de seus familiares ${ }^{3}$.

Indivíduos maiores de 65 anos de idade apresentam taxas de incidência e de mortalidade por câncer superiores aos mais jovens, representando cerca de $70 \%$ dos óbitos por neoplasias. A população idosa é heterogênea, devido às diferentes comorbidades, característica que pode influenciar no tratamento e na mortalidade ${ }^{4}$.

envelhecimento pode interferir no tratamento quimioterápico, pois a senescência acarreta diversas mudanças fisiológicas, aumentando a vulnerabilidade dos tecidos à toxicidade dos antineoplásicos e ocasionando alterações farmacocinéticas, com diminuição da excreção renal dos metabólitos tóxicos, diminuição do volume de distribuição de agentes hidrossolúveis e diminuição da absorção intestinal. Outro fator a ser destacado é que, com o envelhecimento, a biologia tumoral sofre alteração, podendo apresentar diminuição da sensibilidade ao tratamento ${ }^{5}$.

Apesar dos avanços da medicina quanto ao tratamento das neoplasias, o câncer ainda é um estigma de morte e, portanto, seu diagnóstico torna-se amedrontador, sendo geralmente acompanhado de angústia, desesperança, isolamento e temores ${ }^{6}$. Soma-se a isso um tratamento agressivo, principalmente - quimioterápico, com consequências físicas, emocionais e sociais, causando diversas perdas de autonomia no cotidiano ${ }^{7,8}$.

Avaliar a QV de pacientes idosos com neoplasias malignas implica em conhecer melhor os fatores que causam impacto no tratamento, o que ajuda a estabelecer, de forma mais eficiente, os protocolos assistenciais a esse grupo em particular, bem como minimizar os prejuízos causados, levando a uma melhora no bem-estar geral do paciente.

$\bigcirc$ presente estudo teve como objetivos avaliar a QV dos idosos sob tratamento quimioterápico antineoplásico, atendidos em um ambulatório de um hospital de referência do estado do Pará, Brasil, assim como a associação com fatores socioeconômicos, nível de dependência e dados clínicos.

\section{MATERIAIS E MÉTODOS}

Este estudo foi realizado no Hospital Ophir Loyola $(\mathrm{HOL})$, Centro de Alta Complexidade em Oncologia e principal referência em tratamento oncológico da rede pública do estado do Pará.

Realizou-se um estudo epidemiológico transversal observacional, com idosos de ambos os sexos, submetidos à quimioterapia antineoplásica no $\mathrm{HOL}$, no período de abril a setembro de 2015. Excluíram-se do estudo os idosos com algum déficit cognitivo ou distúrbio mental que dificultasse o fornecimento de informações. A identificação de déficits cognitivos ou de distúrbio mental foi realizada pelos pesquisadores no momento do recrutamento ou por informações do prontuário.

- cálculo amostral foi realizado com base no quantitativo efetivo de pacientes submetidos à quimioterapia no ano de 2010 (dados mais recentes disponíveis). Naquele ano, 3.021 pacientes foram submetidos à quimioterapia no $\mathrm{HOL}$, e desses 370 possuíam idade superior a 60 anos. $\bigcirc$ corte de idade definido neste estudo baseou-se no Estatuto do Idoso, Lei $n^{\circ} 10.741 / 2003$, que define como idoso a pessoa com idade igual ou superior a 60 anos $^{9}$. Considerando a população do estudo finita e um erro amostral de $5 \%$, o cálculo foi realizado conforme descrito por Miot $^{10}$. Assim, o tamanho amostral foi estimado em 157 idosos. A amostra foi composta por 200 idosos que aceitaram participar da pesquisa, assinando o Termo de Consentimento Livre e Esclarecido, e que atendiam aos critérios de elegibilidade.

Dados socioeconômicos e clínicos foram obtidos por meio de formulário com questões referentes à idade, sexo, estado civil, procedência, raça, escolaridade, ocupação, renda familiar, contribuição para a renda, número de pessoas com quem reside, religião, tipo de câncer, tempo de tratamento, outros tratamentos, comorbidades e protocolo quimioterápico. $\bigcirc$ perfil clínico-epidemiológico desses participantes foi explorado em estudo publicado previamente $^{11}$.

O nível de dependência funcional foi avaliado pela aplicação da Escala de Lawton e Brody ${ }^{12}$. Esse instrumento permite analisar a capacidade do indivíduo em realizar as atividades instrumentais da vida diária, como utilizar o telefone, administrar o dinheiro, realizar compras, preparar refeições e viajar ${ }^{13}$.

Para avaliar a QV dos participantes, foi utilizado - questionário EORTC QLQ-C30 v3 (European Organization for Research and Treatment of Cancer Quality of Life Questionnaire)14,15, com questões que avaliam os sintomas nas últimas semanas. Foi desenvolvido pela Organização Europeia de Pesquisa e Tratamento do Câncer, sendo atualmente o instrumento mais utilizado no mundo em estudos clínicos com pacientes portadores dessa patologia ${ }^{16}$. É formado por 30 itens que abrangem 15 domínios, divididos em três escalas distintas: escala de estado de saúde global e qualidade de vida (possui apenas 
um domínio, medida global de saúde); escala funcional (domínios função física, desempenho de papéis, função emocional, função cognitiva e função social); e escala de sintomas (domínios fadiga, náusea e vômito, dor, dispneia, insônia, perda de apetite, constipação, diarreia e dificuldade financeira). As escalas de saúde global e funcional indicam melhor QV à medida que sua pontuação se aproxima de 100. Para a escala de sintomas, a análise é inversa, sendo melhor o desempenho para a QV quando os escores aproximam-se da pontuação mínima (zero) ${ }^{17}$.

Para a análise dos dados, foi utilizado o software IBM SPSS Statistics 20. O teste de análise de variância (ANOVA) foi empregado para verificar as associações entre os dados sociodemográficos e clínicos com os escores dos instrumentos utilizados. Foram considerados significativos os fatores com valor de $p \leq 0,05$.

Este estudo foi aprovado em 10 de abril de 2015 pelo Comitê de Ética em Pesquisa do HOL, sob o número 260.422, e cumpriu os princípios éticos contidos na Resolução n ${ }^{\circ}$ 466/2012 do Conselho Nacional de Saúde.

\section{RESULTADOS}

As características sociodemográficas e clínicas dos 200 participantes já foram previamente descritas por Santos et al. ${ }^{11}$, que identificaram: prevalência de indivíduos entre 61 e 80 anos de idade (83,5\%); sem predominância de sexo; $82,5 \%$ possuíam escolaridade até o nível fundamental completo; 79,5\% possuíam renda familiar inferior a três salários mínimos; $57,0 \%$ eram casados ou em união estável; e 87,5\% participavam ativamente na renda familiar. $\bigcirc$ tipo de câncer mais comum identificado foi o de mama $(26,5 \%)$, seguido pelo de próstata $(17,0 \%)$; e os tratamentos antineoplásicos ou adjuvantes mais prevalentes foram bisfosfonato (18,0\%) e agente alquilante $(41,0 \%)$.

Quanto aos resultados do presente estudo, o nível de dependência dos idosos foi identificado pela Escala de Lawton e Brody. Dos 200 idosos investigados, $183(91,5 \%)$ foram classificados como dependentes parciais, $12(6,0 \%)$ como dependentes totais e apenas cinco $(2,5 \%)$ como independentes $\left(p<0,001, \chi^{2}\right.$ de homogeneidade).

A tabela 1 apresenta os resultados da avaliação da QV dos idosos segundo escalas e domínios do EORTC QLQ-C30 v3. Os escores médios das escalas de saúde global e funcional demonstraram que os idosos apresentaram um nível mediano de $Q V$, enquanto a escala de sintomas mostrou níveis de QV mais altos que os das outras escalas.

Para os domínios da escala funcional, que correspondem às funções física, emocional, cognitiva, social e o desempenho de papéis, os escores menores indicam baixa QV. Dessa forma, os domínios que mais influenciaram negativamente foram a função social e o desempenho de papéis. A função cognitiva foi a mais preservada, contribuindo para uma melhor QV.

Tabela 1 - Escores das escalas e domínios do EORTC QLQ-C30 v3 de idosos em tratamento quimioterápico atendidos no HOL em Belém, estado do Pará, Brasil, 2015

EORTC QLQ-C30 v3 Média \pm DP

Escalas

Escala de saúde global

Escala funcional

Escala de sintomas

Domínios

$\begin{array}{ll}\text { Função física } & 54,67 \pm 28,31 \\ \text { Função emocional } & 54,38 \pm 35,33 \\ \text { Função cognitiva } & 69,17 \pm 32,30 \\ \text { Função social } & 47,17 \pm 41,37 \\ \text { Desempenho de papéis } & 42,25 \pm 40,61 \\ \text { Fadiga } & 52,06 \pm 33,17 \\ \text { Náusea e vômito } & 21,83 \pm 26,60 \\ \text { Dor } & 41,75 \pm 38,50 \\ \text { Dispneia } & 21,83 \pm 36,08 \\ \text { Insônia } & 46,67 \pm 43,18 \\ \text { Perda de apetite } & 48,67 \pm 44,06 \\ \text { Constipação } & 28,00 \pm 40,30 \\ \text { Diarreia } & 26,17 \pm 37,79 \\ \text { Dificuldade financeira } & 59,67 \pm 42,61\end{array}$

DP: Desvio padrão.

Escores elevados para os domínios da escala de sintomas indicam que os sintomas estão influenciando negativamente na QV. Os domínios dificuldade financeira e fadiga foram os que mais contribuíram para o declínio da QV dos participantes deste estudo.

No presente estudo, não houve associação estatística significativa $(p>0,05)$ entre as variáveis escolaridade, comorbidades, religião, estado civil e procedência e as escalas e domínios para a QV.

A escala de saúde global demonstrou associação apenas com a variável nível de dependência ( $p<$ 0,001, ANOVA). O elevado nível de dependência relaciona-se com o declínio da percepção de saúde global. Idosos classificados como dependentes totais obtiveram escore médio baixo (20,39 \pm $22,71)$ na escala de saúde global, enquanto pacientes independentes e parcialmente dependentes apresentaram escore médio relativamente alto, 62,50 $( \pm 18,82)$ e $55,59( \pm 24,81)$, respectivamente.

Quanto à escala funcional, observou-se relação estatística significante com as seguintes variáveis: renda familiar ( $p=0,002)$, ocupação $(p=0,021)$, número de pessoas que residem com 0 idoso $(p=0,008)$, necessidade de cuidador $(p=0,013)$, presença de cuidador $(p=0,006)$ e nível de dependência ( $p<$ 0,001) (Tabela 2). 
Tabela 2 - Escala funcional e variáveis estatisticamente significativas de idosos em tratamento quimioterápico atendidos no HOL em Belém, estado do Pará, Brasil, 2015

\begin{tabular}{|c|c|c|c|c|}
\hline Variáveis & $\mathrm{N}$ & Média da escala* & $\pm \mathrm{DP}$ & $p<0,05^{+}$ \\
\hline \multicolumn{5}{|l|}{ Escala funcional } \\
\hline \multicolumn{5}{|c|}{ Renda familiar (salário mínimo) } \\
\hline$<1$ & 12 & 40,19 & $\pm 23,39$ & \multirow{7}{*}{0,002} \\
\hline 1 & 70 & 50,19 & $\pm 26,86$ & \\
\hline 2 & 77 & 55,09 & $\pm 27,11$ & \\
\hline 3 & 25 & 50,13 & $\pm 26,27$ & \\
\hline 4 & 4 & 77,22 & $\pm 17,90$ & \\
\hline 5 & 4 & 80,00 & $\pm 20,69$ & \\
\hline$>5$ & 8 & 81,67 & $\pm 24,36$ & \\
\hline \multicolumn{5}{|l|}{ Ocupação } \\
\hline Atividade urbana & 15 & 70,67 & $\pm 23,14$ & \multirow{3}{*}{0,021} \\
\hline Atividade rural & 6 & 69,26 & $\pm 12,84$ & \\
\hline Sem ocupação & 164 & 52,68 & $\pm 27,68$ & \\
\hline \multicolumn{5}{|c|}{ Número de pessoas com quem mora } \\
\hline 1 & 54 & 58,85 & $\pm 26,05$ & \multirow{6}{*}{0,008} \\
\hline 2 & 41 & 48,18 & $\pm 25,78$ & \\
\hline 3 & 36 & 55,12 & $\pm 28,14$ & \\
\hline 4 & 28 & 62,62 & $\pm 27,87$ & \\
\hline$>4$ & 26 & 38,38 & $\pm 23,67$ & \\
\hline Mora só & 15 & 58,96 & $\pm 30,08$ & \\
\hline \multicolumn{5}{|l|}{ Precisa de cuidador } \\
\hline $\operatorname{Sim}$ & 95 & 48,82 & $\pm 27,63$ & \multirow{2}{*}{0,013} \\
\hline Não & 105 & 58,43 & $\pm 26,50$ & \\
\hline \multicolumn{5}{|l|}{ Possui cuidador } \\
\hline Sim & 124 & 58,01 & $\pm 28,52$ & \multirow{2}{*}{0,006} \\
\hline Não & 76 & 47,11 & $\pm 24,15$ & \\
\hline \multicolumn{5}{|l|}{ Escala de Lawton e Brody } \\
\hline Dependência total & 12 & 18,15 & $\pm 15,51$ & \multirow{3}{*}{$<0,001$} \\
\hline Dependência parcial & 182 & 55,60 & $\pm 26,33$ & \\
\hline Independência & 6 & 72,59 & $\pm 25,83$ & \\
\hline
\end{tabular}

* A média dos escores da escala funcional variam de 0 a 100; quanto maior a pontuação, melhor a QV. ${ }^{\dagger}$ ANOVA. DP: Desvio padrão.

A escala de sintomas apresentou associação estatisticamente significativa com as variáveis faixa etária $(p=0,029)$, presença de cuidador $(p=0,013)$ e nível de dependência $(p=0,006)$ (Tabela 3).

Quando se relacionou as escalas e os domínios de QV ao protocolo de tratamento quimioterápico utilizado, observou-se uma relação estatística significante entre a escala de sintomas e três domínios dessa mesma escala (fadiga, dispneia e perda de apetite). Os pacientes em tratamento com anticorpo monoclonal apresentaram melhor escore de QV na escala de sintomas $(28,49 \pm 19,04 ; p=0,002)$, fadiga $(37,04 \pm 26,06 ; p=0,001)$ e dispneia $(0,00$; $p=0,003)$, enquanto aqueles em uso de bisfosfonato apresentaram melhor escore na variável perda de apetite $(26,85 \pm 39,70 ; p=0,001)$.

Os domínios da escala funcional (funções física, emocional, cognitiva, social e desempenho de papéis) demonstraram associação significativa com as variáveis renda, precisa de cuidador, possui cuidador e nível de dependência, conforme descrito na tabela 4. 
Tabela 3 - Escala de sintomas e variáveis estatisticamente significativas de idosos em tratamento quimioterápico atendidos no HOL em Belém, estado do Pará, Brasil, 2015

\begin{tabular}{|c|c|c|c|c|}
\hline Variáveis & N & Média da escala* & $\pm \mathrm{DP}$ & $p<0,05^{+}$ \\
\hline \multicolumn{5}{|l|}{ Escala de sintomas } \\
\hline \multicolumn{5}{|l|}{ Faixa etária (anos) } \\
\hline$=60$ & 17 & 51,73 & $\pm 20,80$ & \multirow{6}{*}{0,029} \\
\hline $61-70$ & 102 & 42,56 & $\pm 22,69$ & \\
\hline $71-80$ & 65 & 37,36 & $\pm 24,91$ & \\
\hline 81-90 & 14 & 28,75 & $\pm 19,45$ & \\
\hline $91-100$ & 1 & 38,46 & & \\
\hline $101 \pm$ & 1 & 0,00 & & \\
\hline \multicolumn{5}{|l|}{ Precisa de cuidador } \\
\hline Sim & 95 & 44,78 & $\pm 23,77$ & \multirow{2}{*}{0,013} \\
\hline Não & 105 & 36,53 & $\pm 22,76$ & \\
\hline \multicolumn{5}{|l|}{ Escala de Lawton e Brody } \\
\hline Dependência total & 12 & 61,32 & $\pm 23,87$ & \multirow{3}{*}{0,006} \\
\hline Dependência parcial & 182 & 39,15 & $\pm 22,78$ & \\
\hline Independência & 6 & 38,03 & $\pm 30,31$ & \\
\hline
\end{tabular}

* A média dos escores da escala de sintomas variam de 0 a 100; quanto menor a pontuação, melhor a QV. ${ }^{\dagger}$ ANOVA. DP: Desvio padrão.

Tabela 4 - Domínios função física, função emocional, função cognitiva, função social e desempenho de papéis e variáveis estatisticamente significativas de idosos em tratamento quimioterápico atendidos no $\mathrm{HOL}$ em Belém, estado do Pará, Brasil, 2015

\begin{tabular}{|c|c|c|c|c|c|c|}
\hline Variáveis & N & Função física* & Função emocional* & Função cognitiva* & Função social ${ }^{*}$ & $\begin{array}{l}\text { Desempenho de } \\
\text { papéis* }\end{array}$ \\
\hline Renda familiar (salário mínimo) & & $<0,001^{\dagger}$ & $0,031^{\dagger}$ & - & - & $0,006^{+}$ \\
\hline$<1$ & 12 & $42,78 \pm 24,53$ & $37,50 \pm 29,19$ & - & - & $22,22 \pm 35,77$ \\
\hline 1 & 70 & $53,52 \pm 27,94$ & $49,17 \pm 36,40$ & - & - & $39,05 \pm 38,06$ \\
\hline 2 & 77 & $54,72 \pm 27,37$ & $57,36 \pm 33,63$ & - & - & $42,64 \pm 40,87$ \\
\hline 3 & 25 & $45,07 \pm 27,30$ & $51,67 \pm 38,34$ & - & - & $36,00 \pm 42,40$ \\
\hline 4 & 4 & $78,33 \pm 26,32$ & $89,58 \pm 10,49$ & - & - & $66,67 \pm 30,43$ \\
\hline 5 & 4 & $81,67 \pm 28,48$ & $72,92 \pm 32,90$ & - & - & $83,33 \pm 33,33$ \\
\hline$>5$ & 8 & $86,67 \pm 17,82$ & $78,13 \pm 28,85$ & - & - & $83,33 \pm 35,63$ \\
\hline Precisa de cuidador & & $<0,001^{\dagger}$ & - & $0,032^{+}$ & - & - \\
\hline $\operatorname{Sim}$ & 95 & $46,46 \pm 28,62$ & - & $64,04 \pm 33,54$ & - & - \\
\hline Não & 105 & $62,10 \pm 25,99$ & - & $73,81 \pm 30,56$ & - & - \\
\hline Possui cuidador & & - & $0,006^{+}$ & - & $<0,001^{\dagger}$ & $0,024^{\dagger}$ \\
\hline Sim & 124 & - & $59,68 \pm 35,46$ & - & $57,12 \pm 41,38$ & $47,31 \pm 41,56$ \\
\hline Não & 76 & - & $45,72 \pm 33,60$ & - & $30,92 \pm 36,08$ & $33,99 \pm 37,85$ \\
\hline Escala de Lawton e Brody & & $<0,001^{\dagger}$ & $<0,001^{\dagger}$ & $<0,001^{\dagger}$ & $<0,001^{\dagger}$ & $<0,001^{\dagger}$ \\
\hline Dependência total & 12 & $26,67 \pm 26,51$ & $12,50 \pm 16,48$ & $36,11 \pm 33,96$ & $2,78 \pm 9,62$ & $5,56 \pm 19,25$ \\
\hline Dependência parcial & 182 & $56,15 \pm 27,54$ & $56,46 \pm 34,57$ & $70,97 \pm 31,16$ & $49,18 \pm 41,21$ & $43,59 \pm 40,20$ \\
\hline Independência & 6 & $65,56 \pm 28,10$ & $75,00 \pm 30,73$ & $80,56 \pm 30,58$ & $75,00 \pm 25,28$ & $75,00 \pm 41,83$ \\
\hline
\end{tabular}

${ }^{*}$ A média dos escores dos domínios variou de 0 a 100; quanto maior a pontuação, melhor a $Q V .{ }^{\dagger}$ ANOVA. - Não significante (p > 0,05). 


\section{DISCUSSÃO}

Os resultados desta pesquisa concordam com os de Pereira et al. ${ }^{18}$, que ao aplicar a Escala de Lawton e Brody em 112 pacientes oncogeriátricos, internados em um hospital universitário do Pará, identificaram os mesmos como dependentes parciais. No entanto, diferem dos resultados encontrados por Lera et al. ${ }^{7}$, que avaliaram 40 idosos com câncer em tratamento quimioterápico, tendo caracterizado sua amostra como independentes para a Escala de Lawton e Brody. $\bigcirc$ nível de dependência no contexto oncológico indica a preservação ou o declínio da funcionalidade do paciente nas esferas física, psicológica, econômica e social $^{19}$.

O escore para a escala de saúde global encontrados nesta pesquisa $(54,67 \pm 28,31)$ aproxima-se do encontrado por Grutsch et al. ${ }^{20}(51,96 \pm 3,86)$ para os pacientes oncológicos ambulatoriais em quimioterapia de duas cidades dos Estados Unidos. Esses autores apresentaram escores próximos aos deste estudo para os domínios função física $(53,92 \pm 4,12)$, função emocional $(58,99 \pm 5,29)$, função cognitiva $(63,24 \pm$ $4,71)$, desempenho de papéis $(47,06 \pm 5,91)$, insônia $(49,02 \pm 6,17)$ e perda de apetite $(46,08 \pm 6,30)$. Entre os achados de Grutsch et al. ${ }^{20}$, destaca-se ainda a diferença entre os escores dos pacientes do estudo quando comparados àqueles que ainda não haviam iniciado o tratamento quimioterápico e a população em geral, encontrando uma discrepância de até 20 ou 30 pontos entre os grupos, classificando os primeiros como baixos para a QV, o que demonstrou uma possível relação negativa entre a QV e a quimioterapia.

Entretanto, Arnold et al. ${ }^{21}$, que realizaram um estudo multicêntrico observacional com um grupo de pacientes oncológicos em quimioterapia e outro que não aceitou realizar o tratamento, constataram que os escores obtidos na escala de saúde global dos idosos em tratamento eram mantidos durante o curso da quimioterapia, enquanto para os que não se tratavam, esses escores iam decaindo com a evolução da doença; para as escalas de sintomas e funcional, ocorreu queda dos escores em ambos os grupos.

Neste estudo, idosos que apresentaram grande dependência para realizar as atividades instrumentais da vida diária possuíam uma percepção de baixa QV geral. Esse resultado difere do encontrado por Wiltink et al. $^{22}$, que realizaram um estudo com holandeses com idade média de 77 anos e não encontraram associação entre a funcionalidade e a escala de saúde global.

Quando investigada a associação entre a escala funcional e as variáveis analisadas, observou-se escore médio maior $(81,67 \pm 24,36)$ para idosos que tinham uma renda familiar maior que cinco salários mínimos, mostrando melhor QV; e escore médio menor $(40,19$ $\pm 23,39$ ) para rendas menores que um salário mínimo, apresentando menor QV. Para a variável ocupação, idosos que exerciam atividades urbanas obtiveram escore médio maior $(70,67 \pm 23,14)$, mostrando melhor QV; já os que não tinham ocupação apresentaram escore menor $(52,68 \pm 27,68)$, mostrando baixa $Q V$.
Idosos que afirmaram precisar de cuidador obtiveram escore menor $(48,82 \pm 27,63)$, indicando baixa QV; os que declararam possuir um cuidador tiveram escore maior $(58,01 \pm 28,52)$ e, portanto, melhor QV. Os participantes que foram avaliados como independentes obtiveram maior escore médio $(72,59 \pm 25,83)$ na escala funcional, indicando bom desempenho na realização de atividades cotidianas, lazer e práticas sociais, menor estresse emocional e menos alterações na cognição.

Segundo Anjos e Zago 23, a família ou as pessoas próximas realizam a função de cuidadores do idoso dentro do ambiente familiar, que é considerado o melhor local para executar tal função. A situação do idoso em tratamento mostra a necessidade de tal cuidador, devido aos efeitos adversos do mesmo, e, com a perda da capacidade funcional, as exigências aumentam, podendo também causar prejuízos à saúde do cuidador ${ }^{24}$.

Foi identificada a associação entre a escala de sintomas e a variável protocolo quimioterápico. $\bigcirc$ estudo de Garcia et al. ${ }^{25}$, que aplicou o EORTC QLQ-C30 em mulheres com neoplasia de mama, mostrou que o tratamento quimioterápico interfere negativamente na capacidade funcional, diminuindo sua autonomia. Efeitos do tratamento, sintomas como alterações no paladar, perda da força muscular e menor concentração levam à diminuição da QV. Entretanto, os resultados obtidos nesta pesquisa demonstraram elevado desempenho para a QV entre os idosos, com destaque àqueles em tratamento com bisfosfonato e anticorpo monoclonal. Essa divergência pode estar relacionada à classe de medicamento utilizada. A utilização de bisfosfonato, seja individualmente ou em associação com outras classes de antineoplásicos, possui significante efeito positivo na QV dos pacientes ${ }^{26}$.

Neste estudo, o escore do domínio fadiga evidenciou uma interferência moderada do cansaço e da fadiga sobre os idosos e uma relação com o protocolo quimioterápico administrado. Um estudo ${ }^{27}$ que avaliou a fadiga em pacientes submetidos à quimioterapia identificou mais fadiga quando comparado ao grupo controle e mais sintomas depressivos, mesmo depois de decorrido um ano do tratamento. Segundo Buchowski et al. ${ }^{28}$, a fadiga pode ser utilizada como preditor da morbidade e da mortalidade em adultos e é uma das causas da atividade física restrita, mostrando também que há uma correlação entre a fadiga autorrelatada e mudanças no desempenho.

domínio perda de apetite também demonstrou associação estatisticamente significativa com o tipo de protocolo quimioterápico antineoplásico utilizado. Esses medicamentos são associados à alteração do paladar, associação essa que se deve à neurotoxicidade periférica que pode ocasionar neuropatia periférica com consequentes alterações sensitivas; por isso, as alterações no paladar também são relatadas frequentemente por pacientes que não apresentam náuseas ${ }^{29}$. 
A função física demonstrou que os idosos apresentavam dificuldade moderada em realizar atividades físicas e associação entre a renda, visto que pacientes que tinham menores rendimentos mensais apresentavam mais interferências na função física. Em um estudo longitudinal ${ }^{30}$ realizado na Dinamarca a respeito do efeito do câncer de mama sobre a renda, foi observado que a perda de renda em pacientes com câncer pode estar associada aos efeitos negativos que a doença e o tratamento têm sobre o trabalho.

A função emocional mostrou-se alterada, indicando que os pacientes deste estudo apresentaram moderada irritação, tensão, tristeza e preocupação. $\bigcirc$ câncer é uma doença estigmatizante, que provoca grande carga de sofrimento e preocupação para o paciente, aumentando o nível de estresse e gerando mudanças de comportamento ${ }^{31}$. Os pacientes oncológicos tendem a relatar o momento da doença como angustiante e de muita tristeza, e as respostas emocionais mais frequentes apresentadas são ansiedade, raiva e depressão. Menor capacidade de enfrentamento por pacientes em tratamento quimioterápico tem sido associada ao aumento de sintomas do câncer, aumentando o sofrimento físico e psicológico e interferindo na $\mathrm{QV}^{32}$.

A função cognitiva revelou relação com o nível de dependência, visto que idosos classificados com dependência total para as atividades instrumentais da vida diária apresentaram baixa QV. A literatura tem mostrado que a função cognitiva preservada é um fator que contribui para a manutenção da independência do idoso; caso contrário, tem um efeito negativo na capacidade funcional ${ }^{33}$.

\section{CONCLUSÃO}

Este estudo proporcionou avaliar a QV relacionada à saúde dos idosos submetidos a tratamento quimioterápico antineoplásico. Foram encontrados resultados que apontavam uma QV mediana para as escalas de saúde global e funcional; e a escala de sintomas demonstrou uma interferência menor dos efeitos adversos na QV geral. Os domínios mais afetados foram dificuldade financeira, fadiga, desempenho de papéis e função social. A maioria dos idosos foi classificada como dependentes parciais na Escala de Lawton e Brody.

Foi revelada grande relação negativa entre a capacidade de realizar atividades instrumentais da vida diária com todas as escalas de QV. Os déficits funcionais interferem na esfera financeira por limitar a capacidade de trabalhar, aumentam a necessidade de cuidador para auxiliar nas atividades diárias, provocam declínio na participação social e elevam o sofrimento psicológico e físico desses idosos.

Os resultados deste estudo contribuem para a identificação de fatores socioeconômicos e clínicos que interferem na QV dos pacientes oncogeriátricos, ao evidenciar a associação entre a perda da capacidade funcional do idoso com a função emocional, mostrando que a limitação física causada pelo envelhecimento e a doença afetam mais do que o funcionamento biológico do indivíduo.

Dessa forma, a escala EORTC QLQ-C30 v3 mostrou-se um importante instrumento para a equipe de saúde envolvida no cuidado do paciente oncológico, para a avaliação do desempenho físico, psicológico e social do idoso com câncer, permitindo inferir os reais resultados do tratamento quimioterápico antineoplásico no cotidiano do paciente e desenvolver estratégias para promover o bem-estar e a QV.

Com o intuito de identificar a forma com que a doença e seu tratamento afetam o decorrer da vida do idoso, sugere-se a realização de pesquisas longitudinais para avaliar as mudanças na QV e na capacidade funcional durante os períodos de pré-tratamento, tratamento e pós-tratamento.

\section{CONFLITOS DE INTERESSE}

Os autores declaram que não houve conflito de interesses.

\section{CONTRIBUIÇÃO DOS AUTORES}

Todos os autores contribuíram com a idealização do estudo, a análise e a interpretação dos dados e com a redação do manuscrito, aprovando a versão final publicada. Declaram-se responsáveis pelo conteúdo integral do artigo, garantindo sua precisão e integridade.

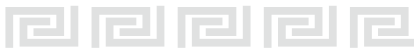

\section{REFERÊNCIAS}

1 Vieira Junior WM, Martins M. Idosos e planos de saúde no Brasil: análise das reclamações recebidas pela Agência Nacional de Saúde Suplementar. Cienc Saude Coletiva. 2015 dez;20(12):3817-26.

2 Moraes SA, Lopes DA, Freitas ICM. Avaliação do efeito independente de doenças crônicas, fatores sociodemográficos e comportamentais sobre a incapacidade funcional em idosos residentes em Ribeirão Preto, SP, 2007 - Projeto EPIDCV. Rev Bras Epidemiol. 2015 out-dez;18(4):757-70.
3 Schramm JMA, Oliveira AF, Leite IC, Valente JG, Gadelha AMJ, Portela MC, et al. Transição epidemiológica e o estudo de carga de doença no Brasil. Cienc Saude Coletiva. 2004 out-dez;9(4):897-908.

4 Antunes YPPV, Bugano DDG, Giglio A, Kaliks RA, Karnakis T, Pontes LB. Características clínicas e de sobrevida global em pacientes oncológicos idosos num centro oncológico terciário. Einstein (São Paulo). 2015 out-dez;13(4):487-91. 
5 Costa F, Barata FJ. Abordagem terapêutica do carcinoma pulmonar de não pequenas células no idoso. Rev Port Pneumol. 2007 nov-dez; 13(6):841-54.

6 Rocha LS, Beuter M, Neves ET, Leite MT, Brondani CM, Perlini NMOG. O cuidado de si de idosos que convivem com câncer em tratamento ambulatorial. Texto Contexto Enferm. 2014 jan-mar;23(1): 29-37.

7 Lera AT, Miranda MC, Trevizan LLB, Antonangelo DV, Zanellato RM, Tateyama LTC, et al. Aplicação do instrumento termômetro de estresse em pacientes idosos com câncer: estudo piloto. Rev Bras Clin Med. 2011 mar-abr;9(2):112-6.

8 Terra FS, Costa AMDD, Damasceno LL, Lima TS, Filipini CB, Leite MAC. Avaliação da qualidade de vida de pacientes oncológicos submetidos à quimioterapia. Rev Bras Clin Med. 2013 abr-jun; 11 (2): $112-7$.

9 Brasil. Lei $n^{\circ}$ 10.741, de 1 de outubro de 2003. Dispõe sobre o Estatuto do Idoso e dá outras providências. Diário Oficial da União, Brasília (DF), 2003 out 3; Seção 1.

10 Miot HA. Tamanho da amostra em estudos clínicos e experimentais. J Vasc Bras. 2011 dez; 10(4):275-8.

11 Santos EGA, Souza JC, Santos ALS, Santos MIPO, Oliveira TNC. Perfil clínico-epidemiológico de idosos submetidos à quimioterapia antineoplásica atendidos em um hospital de referência oncológica do estado do Pará, Brasil. Rev Pan-Amaz Saude. 2017 jun;8(2):47-56.

12 Lawton MP, Brody EM. Assessment of older people: self-maintaining and instrumental activities of daily living. Gerontologist. 1969 Oct;9(3):179-86.

13 Lopes MCBT, Lage JSS, Vancini-Campanharo CR, Okuno MFP, Batista REA. Fatores associados ao comprometimento funcional de idosos internados no serviço de emergência. Einstein (São Paulo). 2015 abr-jun;13(2):209-14.

14 Aaronson NK, Ahmedzai S, Bergman B, Bullinger M, Cull A, Duez NJ, et al. The European Organization for Research and Treatment of Cancer QLQ-C30: a quality-of-life instrument for use in international clinical trials in oncology. J Natl Cancer Inst. 1993 Mar;85(5):365-76.

15 Pais-Ribeiro J, Pinto C, Santos C. Validation study of the Portuguese version of the QLC-C30-V.3. Psic Saude Doenças [Internet]. 2008 [cited 2016 Oct 18];9(1):89-102. Available from: http://www.scielo. mec.pt/scielo.php? script=sci_arttext\&pid $=$ S1 645-0 $0862008000100008 \&$ lng $=$ pt.

16 Silva RC. Qualidade de vida em pacientes com linfoma não Hodgkin durante a quimioterapia em regime ambulatorial: avaliação com o questionário EORTC-QLQ-C30 [dissertação]. Barretos (SP): Hospital de Câncer de Barretos; 2013. 92 p.
17 Correia FR. Tradução, adaptação cultural e validação inicial no Brasil da Palliative Outcome Scale (POS) [dissertação]. Ribeirão Preto (SP): Universidade de São Paulo, Escola de Enfermagem de Ribeirão Preto; 2012. 145 p.

18 Pereira EEB, Santos NB, Sarges ESNF. Avaliação da capacidade funcional do paciente oncogeriátrico hospitalizado. Rev Pan-Amaz Saude. 2014 dez;5(4):37-44.

19 Paula AFM, Ribeiro LHM, D’Elboux MJ, Guariento ME. Avaliação da capacidade funcional, cognição e sintomatologia depressiva em idosos atendidos em ambulatório de Geriatria. Rev Bras Clin Med. 2013 jul-set; 11 (3):212-8.

20 Grutsch JF, Ferrans C, Wood PA, Du-Quiton J, Quiton DFT, Reynolds JL, et al. The association of quality of life with potentially remediable disruptions of circadian sleep/activity rhythms in patients with advanced lung cancer. BMC Cancer. 2011 May; 1 1:193.

21 Arnold DT, Hooper CE, Morley A, White P, Lyburn ID, Searle J, et al. The effect of chemotherapy on health-related quality of life in mesothelioma: results from the SWAMP trial. Br J Cancer. 2015 Mar; 1 12(7): $1183-9$.

22 Wiltink LM, Chen TYT, Nout RA, Kranenbarg EMK, Fiocco M, Laurberg $S$, et al. Health-related quality of life 14 years after preoperative short-term radiotherapy and total mesorectal excision for rectal cancer: report of a multicenter randomised trial. Eur J Cancer. 2014 Sep;50(14):2390-8.

23 Anjos ACY, Zago MMF. Ressignificação da vida do cuidador do paciente idoso com câncer. Rev Bras Enferm. 2014 set-out;67(5):752-8.

24 Fuhrmann AC, Bierhals CCBK, Santos NO, Paskulin LMG. Associação entre a capacidade funcional de idosos dependentes e a sobrecarga do cuidador familiar. Rev Gaucha Enferm. 2015 jan-mar;36(1):14-20.

25 Garcia SN, Jacowski M, Castro GC, Galdino C, Guimarães PRB, Kalinke LP. Os domínios afetados na qualidade de vida de mulheres com neoplasia mamária. Rev Gaucha Enferm. 2015 abr-jun;36(2):89-96.

26 Ruggiero SL, Dodson TB, Assael LA, Landesberg R, Marx RE, Mehrotra B. American Association of Oral and Maxillofacial Surgeons position paper on bisphosphonate-related osteonecrosis of the jaws - 2009 update. J Oral Maxillofac Surg. 2009 May;67(5 Suppl):2-12.

27 Ancoli-Israel S, Liu L, Rissling M, Natarajan L, Neikrug AB, Palmer BW, et al. Sleep, fatigue, depression and circadian activity rhythms in women with breast cancer before and after treatment: a 1 -year longitudinal study. Support Care Cancer. 2014 Sep;22(9):2535-45. 
28 Buchowski MS, Simmons SF, Whitaker LE, Powers J, Beuscher L, Choi L, et al. Fatigability as a function of physical activity energy expenditure in older adults. AGE. 2013 Feb;35(1):179-87.

29 Boltong A, Aranda S, Keast R, Wynne R, Francis PA, Chirgwin J, et al. A prospective cohort study of the effects of adjuvant breast cancer chemotherapy on taste function, food liking, appetite and associated nutritional outcomes. PLoS One. 2014 Jul;9(7):e 103512.

30 Andersen I, Kolodziejczyk C, Thielen K, Heinesen E, Diderichsen F. The effect of breast cancer on personal income three years after diagnosis by cancer stage and education: a register-based cohort study among Danish females. BMC Public Health. 2015 Jan; 15:50.
31 Alves RF, Melo MO, Andrade SFO, Fernandes TS, Gonçalves DL, Freire AA. Qualidade de vida em pacientes oncológicos na assistência em casas de apoio. Aletheia. 2012 mai-dez;38-39:39-54.

32 Tadele N. Evaluation of quality of life of adult cancer patients attending Tikur Anbessa specialized referral hospital, Addis Ababa Ethiopia. Ethiop J Health Sci. 2015 Jan;25(1):53-62.

33 Alexandre TS, Corona LP, Nunes DP, Santos JLF, Duarte YAO, Lebrão ML. Disability in instrumental activities of daily living among older adults: gender differences. Rev Saude Publica. 2014 Jun;48(3):379-89.

Recebido em / Received: 14/8/2017 Aceito em / Accepted: 20/6/2018 